\title{
Spectroscopic, Thermal and Biological Studies on Some Trivalent Ruthenium and Rhodium NS Chelating Thiosemicarbazone Complexes
}

\author{
Vinod K. Sharma, Shipra Srivastava, and Ankita Srivastava \\ Department of Chemistry, Faculty of Science, University of Lucknow, Lucknow 226007, India
}

Received 2 August 2006; Revised 9 November 2006; Accepted 25 December 2006

Recommended by Elena Milaeva

\begin{abstract}
The synthetic, spectroscopic, and biological studies of sixteen ring-substituted 4-phenylthiosemicarbazones and 4-nitrophenylthiosemicarbazones of anisaldehyde, 4-chlorobenzaldehyde, 4-fluorobenzaldehyde, and vanillin with ruthenium(III) and rhodium(III) chlorides are reported here. Their structures were determined on the basis of the elemental analyses, spectroscopic data (IR, electronic, ${ }^{1} \mathrm{H}$ and ${ }^{13} \mathrm{C}$ NMR) along with magnetic susceptibility measurements, molar conductivity and thermogravimetric analyses. Electrical conductance measurement revealed a 1:3 electrolytic nature of the complexes. The resulting colored products are monomeric in nature. On the basis of the above studies, three ligands were suggested to be coordinated to each metal atom by thione sulphur and azomethine nitrogen to form low-spin octahedral complexes with ruthenium(III) while forming diamagnetic complexes with rhodium(III). Both ligands and their complexes have been screened for their bactericidal activities and the results indicate that they exhibit a significant activity.
\end{abstract}

Copyright (c) 2007 Vinod K. Sharma et al. This is an open access article distributed under the Creative Commons Attribution License, which permits unrestricted use, distribution, and reproduction in any medium, provided the original work is properly cited.

\section{INTRODUCTION}

The synthesis and structural investigations of thiosemicarbazones and their metal complexes are of considerable centre of attention because of their potentially beneficial pharmacological properties and a wide variation in their modes of bonding and stereochemistry [1-3]. Coordination chemistry of mixed hard-soft NS donor ligands is a field of current interest. The most important factor in this objective is probably the design of ligands with an appropriate structural backbone. Thiosemicarbazones that are most widely studied are sulphur and nitrogen consisting ligands $[4,5]$. Besides, thiosemicarbazones have emerged as an important sulphur containing ligands in the last two decades [6-9]. The real impetus towards coordination chemistry is the wide range of biological properties depending on parent aldehyde or ketone including antitumour $[10,11]$, antibacterial, and antifungal $[12,13]$ properties as well as their physicochemical effects $[14,15]$. In addition of this, they have been screened for their medicinal properties because they possess some cytotoxic effect. They also stabilize uncommon oxidation states, gen- erate a different coordination number in transition metal complexes in order to participate in various redox reactions $[16,17]$. It is well known that several metal ions enhance and modify the biological activities of thiosemicarbazones, the new metals to such a list are ruthenium [18] and rhodium [19]. Much attention has been drawn towards the chemistry of ruthenium $[20,21]$ and rhodium [22] in different coordination spheres. Due to different oxidation states of ruthenium and rhodium their reactivity depends upon stability of oxidation states. In view of this ruthenium(III), thiosemicarbazones with nitrogen and sulphur as donor atoms have been found to be very efficient catalysts in the oxidation of alcohols and alkenes [23]. With the growing interest of thiosemicarbazones of ruthenium and rhodium metal ions, here we report the synthesis, characterization, and biological activities of the ruthenium(III) and rhodium(III) thiosemicarbazones obtained by condensation of the ring-substituted 4-phenylthiosemicarbazide and 4-nitrophenylthiosemicarbazide with anisaldehyde, 4chlorobenzaldehyde, 4-fluorobenzaldehyde, and vanillin. Biological activities of the complexes and ligands have also been carried out against bacteria Bacillus subtilis and Pseudomonos 


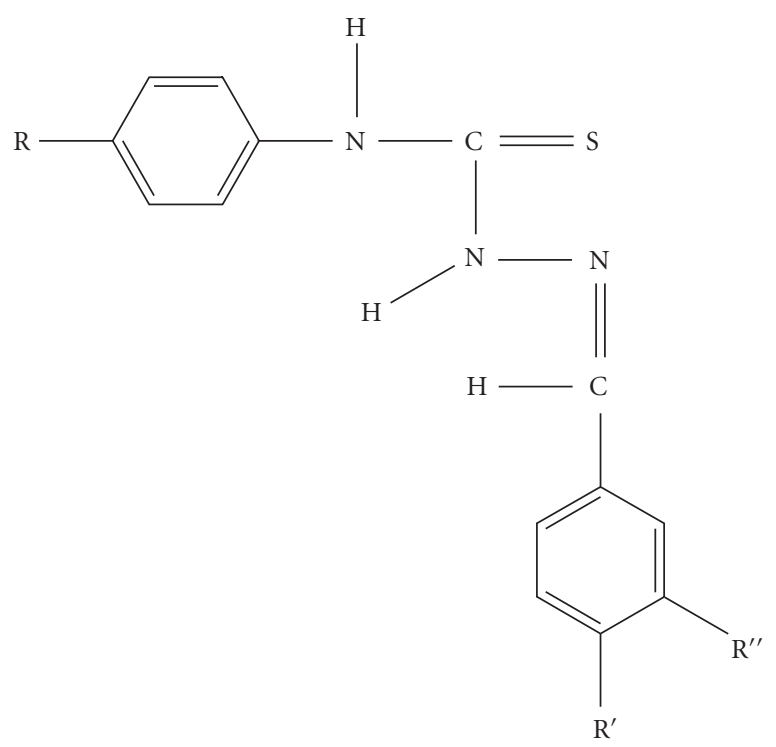

\begin{tabular}{|c|c|c|c|}
\hline $\mathrm{R}$ & $\mathrm{R}^{\prime}$ & $\mathrm{R}^{\prime \prime}$ & Abbreviation \\
\hline $\mathrm{H}$ & $\mathrm{OCH}_{3}$ & $\mathrm{H}$ & HAPT \\
\hline $\mathrm{NO}_{2}$ & $\mathrm{OCH}_{3}$ & $\mathrm{H}$ & HANPT \\
\hline $\mathrm{H}$ & $\mathrm{Cl}$ & $\mathrm{H}$ & HCBPT \\
\hline $\mathrm{NO}_{2}$ & $\mathrm{Cl}$ & $\mathrm{H}$ & HCBNPT \\
\hline $\mathrm{H}$ & $\mathrm{F}$ & $\mathrm{H}$ & HFBPT \\
\hline $\mathrm{NO}_{2}$ & $\mathrm{~F}$ & $\mathrm{H}$ & HFBNPT \\
\hline $\mathrm{H}$ & $\mathrm{OH}$ & $\mathrm{OCH}_{3}$ & HVPT \\
\hline $\mathrm{NO}_{2}$ & $\mathrm{OH}$ & $\mathrm{OCH}_{3}$ & HVNPT \\
\hline
\end{tabular}

FIgURE 1: Structure of the thiosemicarbazone ligands.

aeruginosa in vitro. The ligands used in the study are of the type depicted in Figure 1.

\section{EXPERIMENTAL}

\subsection{Materials}

$\mathrm{RuCl}_{3} \cdot 3 \mathrm{H}_{2} \mathrm{O}$ and $\mathrm{RhCl}_{3} \cdot 3 \mathrm{H}_{2} \mathrm{O}$ and other chemicals were purchased from Merck and Loba chemie, Bombay, India, and were used without further purification.

The antibacterial activity of the ligands and their complexes were tested by using paper disc diffusion method [24] against Bacillus subtilis and Pseudomonas aeruginosa. Nutrient agar medium was prepared by using peptone, beef extract, $\mathrm{NaCl}$, agar-agar, distilled water, and $5 \mathrm{~mm}$ diameter paper discs (whatman No.1) were used. The test organisms were dissolved in ethanol to a concentrations of 1000 and $500 \mathrm{ppm}$ and soaked in filter paper discs of $5 \mathrm{~mm}$ diameter and $1 \mathrm{~mm}$ in thickness. These paper discs were kept in a petri dishes (well sterilized) previously seeded with test organisms. The plates were incubated for $24-30$ hours at $28 \pm 2^{\circ} \mathrm{C}$. The zone of inhibition was calculated in mm carefully. Streptomycin was used as standard. The composition of test media is the factor, which often exerts the greatest effect upon the drug activity. This is particularly true in the case of thiosemicarbazones.

\subsection{Synthesis of the ligands}

4-phenylthiosemicarbazide and 4-nitrophenylthiosemicarbazide were prepared from the appropriate amines by using a standard method [25]. The thiosemicarbazone ligands (Figure 1) were prepared by equimolar quantities of $0.02 \mathrm{~mol}$ of each anisaldehyde ( $2.72 \mathrm{~g})$, 4-chlorobenzaldehyde $(2.80 \mathrm{~g})$, 4-fluorobenzaldehyde $(2.48 \mathrm{~g})$ and vanillin $(3.04 \mathrm{~g})$ in $10 \mathrm{~mL}$ ethanol with an ethanolic solution $(25 \mathrm{~mL})$ of 4 phenyl thiosemicarbazide $(3.34 \mathrm{~g}, 0.02 \mathrm{~mol}) / 4$-nitrophenylthiosemicarbazide ( $4.24 \mathrm{~g}, 0.02 \mathrm{~mol})$. The reaction mixtures were then refluxed on a water bath for 1 hour. Few drops of acetic acid were added during reflux. As precipitate appeared, the reaction mixture was allowed to reflux more along with stirring for 2 hours. The residue formed was separated out, filtered off, washed several times with water, recrystallized from ethanol, and finally dried in vacuo over fused calcium chloride. The proposed chemical structures of the thiosemicarbazone ligands are known to be in good agreement with the ratios concluded from analytical data (see Table 1).

\subsection{Synthesis of the complexes}

$\left[\mathrm{M}(\mathrm{L})_{3}\right] \mathrm{Cl}_{3}(\mathrm{M}=\mathrm{Ru}(\mathrm{III}), \mathrm{Rh}(\mathrm{III}) ; \mathrm{L}=\mathrm{HAPT}, \mathrm{HCBPT}$, HFBPT and HVPT)

Hydrated $\mathrm{RuCl}_{3}(0.261 \mathrm{~g}, 0.001 \mathrm{~mol})$ and $\mathrm{RhCl}_{3}(0.263 \mathrm{~g}$, $0.001 \mathrm{~mol})$ in ethanol $(10 \mathrm{~mL})$ were heated, then metal trichloride solution was suspended in $0.003 \mathrm{~mol}$ of each ligand viz. HAPT (2.56g), HCBPT (2.60 g), HFBPT (2.45 g), and HVPT $(2.70 \mathrm{~g})$ in ethanol $(20 \mathrm{~mL})$. The reaction mixtures were refluxed for 9-12 hours. The precipitates formed were cooled, filtered off, and washed with hot water, hot ethanol, and finally with diethyl ether, and dried in vacuo over fused calcium chloride. The yields were $60-70 \%$.

$\left[\mathrm{M}(\mathrm{L})_{3}\right] \mathrm{Cl}_{3}(\mathrm{M}=\mathrm{Ru}(\mathrm{III}), \mathrm{Rh}(\mathrm{III}) ; \mathrm{L}=\mathrm{HANPT}, \mathrm{HCBNPT}$, HFBNPT and HVNPT)

Hydrated $\mathrm{RuCl}_{3}(0.261 \mathrm{~g}, 0.001 \mathrm{~mol})$ and $\mathrm{RhCl}_{3}(0.263 \mathrm{~g}$, $0.001 \mathrm{~mol})$ in ethanol $(10 \mathrm{~mL})$ were suspended in $20 \mathrm{~mL}$ ethanolic solution of the ligands viz. HANPT $(2.97 \mathrm{~g})$, HCBNPT (3.00 g), HFBNPT (2.86 g), and HVNPT (3.11 g). The reaction mixtures were heated for few minutes, one equivalent of ethanolic solution of the $\mathrm{NaOH}$ was added and the reaction mixtures were refluxed for 9-10 hours. The compound, which was precipitated out, was filtered off, washed with water, cold ethanol, and diethyl ether, and dried in vacuo over fused calcium chloride. The yields were $60-76 \%$.

\subsection{Analyses}

Microanalyses were performed at Elementar Vario III Carlo Erba 1108 in Central Drug Research Institute, Lucknow, India. IR spectra of the ligands and their complexes have been 
TABle 1: Analytical data for the ligands and their $\mathrm{Ru}(\mathrm{III})$ and $\mathrm{Rh}(\mathrm{III})$ complexes.

\begin{tabular}{|c|c|c|c|c|c|c|c|c|c|c|}
\hline \multirow{2}{*}{ Compounds } & \multirow{2}{*}{$\begin{array}{l}\text { M. wt. } \\
\text { found } \\
\text { (calcd.) }\end{array}$} & \multirow{2}{*}{ Yield (\%) } & \multirow{2}{*}{ Color } & \multicolumn{6}{|c|}{ Analysis: found (calcd.)\% } & \multirow{2}{*}{$\mu_{\mathrm{eff}} \mathrm{BM}$} \\
\hline & & & & $\mathrm{C}$ & $\mathrm{H}$ & $\mathrm{N}$ & $\mathrm{Cl} / \mathrm{F}$ & S & M & \\
\hline HAPT & $\begin{array}{c}285 \\
(285)\end{array}$ & 70 & Pale yellow & $\begin{array}{c}63.0 \\
(63.1)\end{array}$ & $\begin{array}{c}5.0 \\
(5.2)\end{array}$ & $\begin{array}{c}14.2 \\
(14.7)\end{array}$ & - & $\begin{array}{c}11.0 \\
(11.2)\end{array}$ & - & - \\
\hline HANPT & $\begin{array}{c}328 \\
(330)\end{array}$ & 72 & Yellow brown & $\begin{array}{c}54.3 \\
(54.5)\end{array}$ & $\begin{array}{c}3.9 \\
(4.2)\end{array}$ & $\begin{array}{c}16.2 \\
(16.9)\end{array}$ & - & $\begin{array}{c}9.5 \\
(9.6)\end{array}$ & - & - \\
\hline НCBPT & $\begin{array}{c}288 \\
(289)\end{array}$ & 70 & Cream yellow & $\begin{array}{c}57.8 \\
(58.1)\end{array}$ & $\begin{array}{c}3.9 \\
(4.1)\end{array}$ & $\begin{array}{c}14.0 \\
(14.5)\end{array}$ & $\begin{array}{c}12.0 \\
(12.1)\end{array}$ & $\begin{array}{c}11.0 \\
(11.0)\end{array}$ & - & - \\
\hline HCBNPT & $\begin{array}{c}332 \\
(334)\end{array}$ & 75 & Yellow & $\begin{array}{c}49.0 \\
(50.2)\end{array}$ & $\begin{array}{c}3.0 \\
(3.2)\end{array}$ & $\begin{array}{c}16.2 \\
(16.7)\end{array}$ & $\begin{array}{c}10.0 \\
(10.4)\end{array}$ & $\begin{array}{c}9.3 \\
(9.5)\end{array}$ & - & - \\
\hline HFBPT & $\begin{array}{c}271 \\
(273)\end{array}$ & 74 & Yellow & $\begin{array}{c}61.0 \\
(61.4)\end{array}$ & $\begin{array}{c}4.0 \\
(4.3)\end{array}$ & $\begin{array}{c}15.0 \\
(15.3)\end{array}$ & $\begin{array}{c}6.2 \\
(6.9)\end{array}$ & $\begin{array}{c}11.4 \\
(11.7)\end{array}$ & - & - \\
\hline HFBNPT & $\begin{array}{c}317 \\
(318)\end{array}$ & 75 & Yellow & $\begin{array}{c}52.6 \\
(52.8)\end{array}$ & $\begin{array}{c}3.0 \\
(3.4)\end{array}$ & $\begin{array}{c}17.2 \\
(17.6)\end{array}$ & $\begin{array}{c}5.4 \\
(5.9)\end{array}$ & $\begin{array}{c}10.0 \\
(10.0)\end{array}$ & - & - \\
\hline HVPT & $\begin{array}{c}300 \\
(301)\end{array}$ & 75 & Yellow brown & $\begin{array}{c}59.1 \\
(59.7)\end{array}$ & $\begin{array}{c}4.7 \\
(4.9)\end{array}$ & $\begin{array}{c}13.8 \\
(13.9)\end{array}$ & - & $\begin{array}{c}10.4 \\
(10.6)\end{array}$ & - & - \\
\hline HVNPT & $\begin{array}{c}345 \\
(346)\end{array}$ & 75 & Yellow & $\begin{array}{c}51.5 \\
(51.9)\end{array}$ & $\begin{array}{c}3.8 \\
(4.0)\end{array}$ & $\begin{array}{c}15.9 \\
(16.1)\end{array}$ & - & $\begin{array}{c}9.2 \\
(9.2)\end{array}$ & - & - \\
\hline$\left[\mathrm{Ru}(\mathrm{HAPT})_{3}\right] \mathrm{Cl}_{3}$ & $\begin{array}{c}1062 \\
(1063)\end{array}$ & 68 & Greenish brown & $\begin{array}{c}50.2 \\
(50.7)\end{array}$ & $\begin{array}{c}3.9 \\
(4.2)\end{array}$ & $\begin{array}{c}11.6 \\
(11.8)\end{array}$ & $\begin{array}{c}9.6 \\
(9.8)\end{array}$ & $\begin{array}{c}9.0 \\
(9.0)\end{array}$ & $\begin{array}{c}9.2 \\
(9.5)\end{array}$ & 1.08 \\
\hline$\left[\mathrm{Ru}(\mathrm{HANPT})_{3}\right] \mathrm{Cl}_{3}$ & $\begin{array}{c}1196 \\
(1197)\end{array}$ & 60 & Black & $\begin{array}{c}44.9 \\
(45.0)\end{array}$ & $\begin{array}{c}3.2 \\
(3.5)\end{array}$ & $\begin{array}{c}13.8 \\
(14.0)\end{array}$ & $\begin{array}{c}8.5 \\
(8.7)\end{array}$ & $\begin{array}{c}8.0 \\
(7.9)\end{array}$ & $\begin{array}{c}8.2 \\
(8.4)\end{array}$ & 1.88 \\
\hline$\left[\mathrm{Ru}(\mathrm{HCBPT})_{3}\right] \mathrm{Cl}_{3}$ & $\begin{array}{c}1075 \\
(1076)\end{array}$ & 60 & Brown & $\begin{array}{c}46.4 \\
(46.8)\end{array}$ & $\begin{array}{c}3.0 \\
(3.3)\end{array}$ & $\begin{array}{c}11.2 \\
(11.7)\end{array}$ & $\begin{array}{c}19.3 \\
(19.5)\end{array}$ & $\begin{array}{c}8.9 \\
(8.6)\end{array}$ & $\begin{array}{c}9.0 \\
(9.3)\end{array}$ & 1.89 \\
\hline$\left[\mathrm{Ru}(\mathrm{HCBNPT})_{3}\right] \mathrm{Cl}_{3}$ & $\begin{array}{c}1210 \\
(1205)\end{array}$ & 62 & Black & $\begin{array}{c}41.3 \\
(41.6)\end{array}$ & $\begin{array}{c}2.5 \\
(2.7)\end{array}$ & $\begin{array}{c}13.2 \\
(13.8)\end{array}$ & $\begin{array}{c}17.1 \\
(17.3)\end{array}$ & $\begin{array}{c}7.9 \\
(7.6)\end{array}$ & $\begin{array}{c}8.0 \\
(8.3)\end{array}$ & 1.78 \\
\hline$\left[\mathrm{Ru}(\mathrm{HFBPT})_{3}\right] \mathrm{Cl}_{3}$ & $\begin{array}{c}1026 \\
(1027)\end{array}$ & 60 & Dark brown & $\begin{array}{c}48.0 \\
(49.1)\end{array}$ & $\begin{array}{c}2.6 \\
(3.5)\end{array}$ & $\begin{array}{c}12.0 \\
(12.2)\end{array}$ & $\begin{array}{c}10.0 \\
(10.2)\end{array}$ & $\begin{array}{c}9.3 \\
(9.3)\end{array}$ & $\begin{array}{c}9.2 \\
(9.8)\end{array}$ & 1.80 \\
\hline$\left[\mathrm{Ru}(\mathrm{HFBNPT})_{3}\right] \mathrm{Cl}_{3}$ & $\begin{array}{c}1160 \\
(1162)\end{array}$ & 60 & Black & $\begin{array}{c}43.1 \\
(43.4)\end{array}$ & $\begin{array}{c}2.6 \\
(2.8)\end{array}$ & $\begin{array}{c}14.2 \\
(14.4)\end{array}$ & $\begin{array}{c}8.8 \\
(9.0)\end{array}$ & $\begin{array}{c}8.2 \\
(8.0)\end{array}$ & $\begin{array}{c}8.4 \\
(8.6)\end{array}$ & 1.90 \\
\hline$\left[\mathrm{Ru}(\mathrm{HVPT})_{3}\right] \mathrm{Cl}_{3}$ & $\begin{array}{c}1110 \\
(1111)\end{array}$ & 60 & Brown & $\begin{array}{c}48.2 \\
(48.6)\end{array}$ & $\begin{array}{c}3.7 \\
(4.0)\end{array}$ & $\begin{array}{c}11.1 \\
(11.3)\end{array}$ & $\begin{array}{c}9.2 \\
(9.4)\end{array}$ & $\begin{array}{c}8.6 \\
(8.5)\end{array}$ & $\begin{array}{c}8.9 \\
(9.0)\end{array}$ & 1.68 \\
\hline$\left[\mathrm{Ru}(\mathrm{HVNPT})_{3}\right] \mathrm{Cl}_{3}$ & $\begin{array}{c}1243 \\
(1245)\end{array}$ & 65 & Black & $\begin{array}{c}43.0 \\
(43.3)\end{array}$ & $\begin{array}{c}3.1 \\
(3.3)\end{array}$ & $\begin{array}{c}13.2 \\
(13.4)\end{array}$ & $\begin{array}{c}8.2 \\
(8.4)\end{array}$ & $\begin{array}{c}7.7 \\
(7.2)\end{array}$ & $\begin{array}{c}7.9 \\
(8.1)\end{array}$ & 1.70 \\
\hline$\left[\mathrm{Rh}(\mathrm{HAPT})_{3}\right] \mathrm{Cl}_{3}$ & $\begin{array}{c}1063 \\
(1065)\end{array}$ & 70 & Orange brown & $\begin{array}{c}50.2 \\
(50.7)\end{array}$ & $\begin{array}{c}3.9 \\
(4.2)\end{array}$ & $\begin{array}{c}11.2 \\
(11.8)\end{array}$ & $\begin{array}{c}9.4 \\
(9.8)\end{array}$ & $\begin{array}{c}9.0 \\
(8.9)\end{array}$ & $\begin{array}{c}9.0 \\
(9.5)\end{array}$ & - \\
\hline$\left[\mathrm{Rh}(\mathrm{HANPT})_{3}\right] \mathrm{Cl}_{3}$ & $\begin{array}{c}1196 \\
(1198)\end{array}$ & 65 & Maroon & $\begin{array}{c}44.8 \\
(45.0)\end{array}$ & $\begin{array}{c}3.3 \\
(3.5)\end{array}$ & $\begin{array}{c}13.9 \\
(14.0)\end{array}$ & $\begin{array}{c}8.5 \\
(8.7)\end{array}$ & $\begin{array}{c}8.0 \\
(8.0)\end{array}$ & $\begin{array}{c}8.2 \\
(8.5)\end{array}$ & - \\
\hline$\left[\mathrm{Rh}(\mathrm{HCBPT})_{3}\right] \mathrm{Cl}_{3}$ & $\begin{array}{c}1076 \\
(1077)\end{array}$ & 60 & Brown & $\begin{array}{c}46.5 \\
(46.7)\end{array}$ & $\begin{array}{c}3.0 \\
(3.3)\end{array}$ & $\begin{array}{c}11.2 \\
(11.6)\end{array}$ & $\begin{array}{c}19.2 \\
(19.4)\end{array}$ & $\begin{array}{c}8.9 \\
(8.4)\end{array}$ & $\begin{array}{c}9.3 \\
(9.5)\end{array}$ & - \\
\hline$\left[\mathrm{Rh}(\mathrm{HCBNPT})_{3}\right] \mathrm{Cl}_{3}$ & $\begin{array}{c}1211 \\
(1213)\end{array}$ & 65 & Brown & $\begin{array}{c}41.2 \\
(41.5)\end{array}$ & $\begin{array}{c}2.4 \\
(2.7)\end{array}$ & $\begin{array}{c}13.3 \\
(13.8)\end{array}$ & $\begin{array}{c}17.1 \\
(17.3)\end{array}$ & $\begin{array}{c}7.9 \\
(7.4)\end{array}$ & $\begin{array}{c}8.2 \\
(8.4)\end{array}$ & - \\
\hline$\left[\mathrm{Rh}(\mathrm{HFBPT})_{3}\right] \mathrm{Cl}_{3}$ & $\begin{array}{c}1028 \\
(1028)\end{array}$ & 62 & Rusty brown & $\begin{array}{c}48.9 \\
(49.0)\end{array}$ & $\begin{array}{c}3.1 \\
(3.5)\end{array}$ & $\begin{array}{c}11.8 \\
(12.2)\end{array}$ & $\begin{array}{c}10.0 \\
(10.2)\end{array}$ & $\begin{array}{c}9.3 \\
(9.0)\end{array}$ & $\begin{array}{c}9.8 \\
(10.0)\end{array}$ & - \\
\hline
\end{tabular}


TABLE 1: continued.

\begin{tabular}{|c|c|c|c|c|c|c|c|c|c|c|}
\hline \multirow[b]{2}{*}{ Compounds } & \multirow{2}{*}{$\begin{array}{l}\text { M. wt. } \\
\text { Found } \\
\text { (Calcd.) }\end{array}$} & \multirow[b]{2}{*}{ Yield (\%) } & \multirow[b]{2}{*}{ Color } & \multicolumn{6}{|c|}{ Analysis: Found (Calcd.)\% } & \multirow[b]{2}{*}{$\mu_{\text {eff }} \mathrm{BM}$} \\
\hline & & & & $\mathrm{C}$ & $\mathrm{H}$ & $\mathrm{N}$ & $\mathrm{Cl} / \mathrm{F}$ & S & M & \\
\hline$\left[\mathrm{Rh}(\mathrm{HFBNPT})_{3}\right] \mathrm{Cl}_{3}$ & $\begin{array}{c}1162 \\
(1163)\end{array}$ & 62 & Brown & $\begin{array}{c}43.1 \\
(43.3)\end{array}$ & $\begin{array}{c}2.6 \\
(2.8)\end{array}$ & $\begin{array}{c}14.3 \\
(14.4)\end{array}$ & $\begin{array}{c}8.9 \\
(9.0)\end{array}$ & $\begin{array}{c}8.2 \\
(8.1)\end{array}$ & $\begin{array}{c}8.6 \\
(8.7)\end{array}$ & - \\
\hline$\left[\mathrm{Rh}(\mathrm{HVPT})_{3}\right] \mathrm{Cl}_{3}$ & $\begin{array}{c}1112 \\
(1113)\end{array}$ & 60 & Maroon & $\begin{array}{c}48.4 \\
(48.5)\end{array}$ & $\begin{array}{c}3.9 \\
(4.0)\end{array}$ & $\begin{array}{c}11.2 \\
(11.3)\end{array}$ & $\begin{array}{c}9.3 \\
(9.4)\end{array}$ & $\begin{array}{c}8.6 \\
(8.3)\end{array}$ & $\begin{array}{c}9.0 \\
(9.1)\end{array}$ & - \\
\hline$\left[\mathrm{Rh}(\mathrm{HVNPT})_{3}\right] \mathrm{Cl}_{3}$ & $\begin{array}{c}1244 \\
(1245)\end{array}$ & 68 & Blackish brown & $\begin{array}{c}43.1 \\
(43.3)\end{array}$ & $\begin{array}{c}3.2 \\
(3.3)\end{array}$ & $\begin{array}{c}13.2 \\
(13.4)\end{array}$ & $\begin{array}{c}8.2 \\
(8.4)\end{array}$ & $\begin{array}{c}7.7 \\
(7.2)\end{array}$ & $\begin{array}{c}8.0 \\
(8.1)\end{array}$ & - \\
\hline
\end{tabular}

recorded in $\mathrm{KBr}$ pellets at Shimadzu FTIR 8201 spectrophotometer in $4000-200 \mathrm{~cm}^{-1}$. Electronic spectra of the complexes were recorded in $\mathrm{CHCl}_{3}$ with a Perkin Elmer Lambda $15 \mathrm{UV} /$ Vis spectrophotometer. ${ }^{1} \mathrm{H}$ and ${ }^{13} \mathrm{C}$ NMR were obtained with a Bruker DRX 300 spectrometer in $\mathrm{CDCl}_{3}$ using TMS as standard. Sulphur was estimated gravimetrically as $\mathrm{BaSO}_{4}$. The percentage of nitrogen was estimated by Kjeldahl method. Magnetic susceptibility measurements on powder form of the complexes were recorded with a Gouy's balance by using mercuric tetrathiocyanato cobaltate(II) as a calibrant at $25^{\circ} \mathrm{C}$. Molar conductance was carried out in $10^{-3} \mathrm{M}$ solution of DMF. Thermogravimetric analyses were obtained at $10^{\circ} \mathrm{C} \mathrm{min}^{-1}$ in the $25-750^{\circ} \mathrm{C}$ using a Shimadzu TGA-50 $\mathrm{H}$ analyzer. A standard method was used for determining metal ions and chlorides volumetrically and gravimetrically [26].

\section{RESULTS AND DISCUSSION}

The complexes were synthesized by reacting ligands with metal ions in $3: 1$ molar ratio in ethanolic medium. Thiosemicarbazones were expected to behave as a bidentate with sulphur and nitrogen as donor atoms or coordination sites (see Figure 2). The present thiosemicarbazone ligands exist as the thione form since it has $-\mathrm{NH}-\mathrm{C}=\mathrm{S}$ thioamide group; although, in many instances, thiol form or equilibrium mixture of both forms has been observed in thiosemicarbazones. All the ruthenium(III) complexes being $\mathrm{d}^{5}$ (low spin), $\mathrm{S}=$ $1 / 2$ behave as paramagnetic and rhodium(III) complexes being $\mathrm{d}^{6}$ (low spin), $\mathrm{S}=0$ act as diamagnetic. The analytical data, magnetic susceptibility, and spectral analyses agree well with the proposed composition of formed complexes. All the complexes have shown good solubility in all the common organic solvents but were found insoluble in ether, water, acetone, and benzene. All the complexes are amorphous powder, stable at room temperature and do not show any decomposition on standing for several months. The molar conductance of the complexes in DMF lies in the range 280$315 \Omega^{-1} \mathrm{~cm}^{2} \mathrm{~mol}^{-1}$ indicating their electrolytic behavior and confirms the ionic nature of the chloride ion. Thus the complexes may be formulated as $\left[\mathrm{M}(\mathrm{L})_{3}\right] \mathrm{Cl}_{3}$ (where $\mathrm{M}=\mathrm{Ru}(\mathrm{III}$ ), Rh(III); L = HAPT, HANPT, HCBPT, HCBNPT, HFBPT, HFBNPT, HVPT, and HVNPT).

The presence of chloride ions in outer sphere was tested both qualitatively and quantitatively and found very positive.

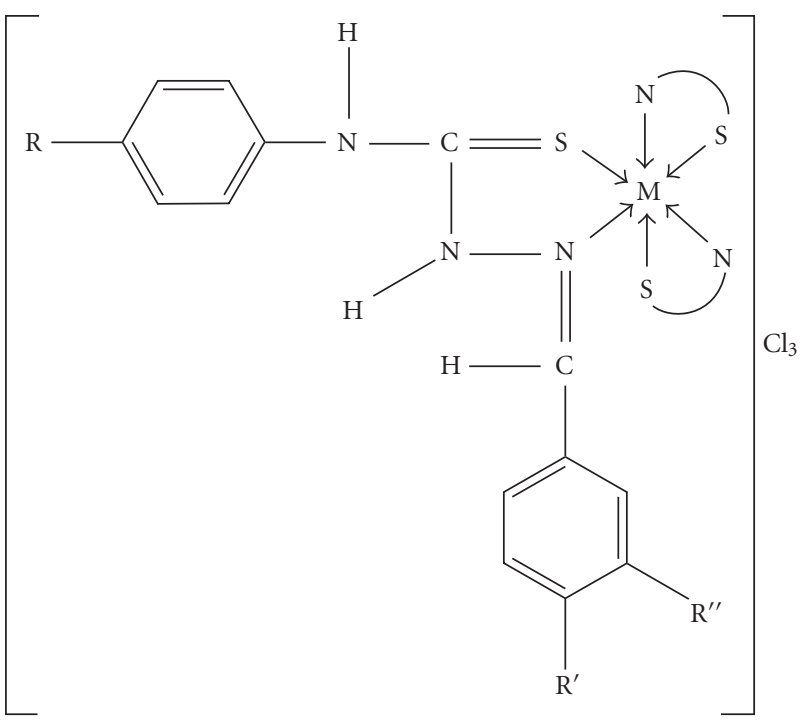

$\mathrm{M}=\mathrm{Ru}(\mathrm{III})$ or $\mathrm{Rh}(\mathrm{III})$

FIGURE 2: Suggested structure of the complexes.

\section{INFRARED SPECTRA}

The tentative infrared absorption frequencies of the ligands and their metal complexes along with their assignments are listed in Table 2. The ligands can act either in keto or in enolic form, depending upon the conditions (e.g., $\mathrm{pH}$ of the medium, oxidation state of the metal ion). All physicochemical properties of the complexes support bidentate chelation of the ligands by the azomethine nitrogen and by thione sulphur. This fact was further supported by the bands including azomethine nitrogen $\nu(\mathrm{C}=\mathrm{N})$ at $1610-1594 \mathrm{~cm}^{-1}$ in ligands and the lowering of this band in complexes results in chelation of the nitrogen to metal ion $[27,28]$. A medium band at $1030-1020 \mathrm{~cm}^{-1}$ which is assigned to $\nu(\mathrm{N}-\mathrm{N})$ in ligands is shifted to the higher frequency in the spectra of all complexes. This kind of shift on hydrazinic nitrogen described the presence of electron withdrawing substituents [29]. However, in metal complexes the band shifts to higher wave number and splits, which is probably the result of the increase in the multiplicity of the $\mathrm{C}-\mathrm{N}$ bond. A strong band 
TABLE 2: Infrared spectral data $\left(\mathrm{cm}^{-1}\right)$ of the ligands and its complexes. $\mathrm{s}=$ strong, $\mathrm{m}=$ medium, $\mathrm{w}=$ weak.

\begin{tabular}{|c|c|c|c|c|c|c|}
\hline \multirow{2}{*}{ Compounds } & \multicolumn{6}{|c|}{ Assignments } \\
\hline & $\nu(\mathrm{N}-\mathrm{H})$ & $\nu(\mathrm{N}-\mathrm{N})$ & $\nu(\mathrm{C}=\mathrm{N})$ & $\nu(\mathrm{C}=\mathrm{S})$ & $\nu(\mathrm{M}-\mathrm{N})$ & $v(\mathrm{M}-\mathrm{S})$ \\
\hline HAPT & $2831 \mathrm{~s}$ & $1030 \mathrm{~m}$ & $1594 \mathrm{~s}$ & $827 \mathrm{~s}$ & - & - \\
\hline HANPT & $2832 \mathrm{~s}$ & $1022 \mathrm{~m}$ & $1600 s$ & $860 \mathrm{~s}$ & - & - \\
\hline НСBРТ & $2830 \mathrm{~s}$ & $1020 \mathrm{~m}$ & $1594 \mathrm{~s}$ & $862 \mathrm{~s}$ & - & - \\
\hline HCBNPT & $2835 \mathrm{~s}$ & $1028 \mathrm{~m}$ & $1595 \mathrm{~s}$ & $872 \mathrm{~s}$ & - & - \\
\hline HFBPT & $2840 \mathrm{~s}$ & $1030 \mathrm{~m}$ & $1610 s$ & $830 \mathrm{~s}$ & - & - \\
\hline HFBNPT & $2835 \mathrm{~s}$ & $1020 \mathrm{~m}$ & $1595 \mathrm{~s}$ & $870 s$ & - & - \\
\hline HVPT & $2842 \mathrm{~s}$ & $1026 \mathrm{~m}$ & $1605 s$ & $860 \mathrm{~s}$ & - & - \\
\hline HVNPT & $2840 \mathrm{~s}$ & $1022 \mathrm{~m}$ & $1610 s$ & $830 \mathrm{~s}$ & - & - \\
\hline$\left[\mathrm{Ru}(\mathrm{HAPT})_{3}\right] \mathrm{Cl}_{3}$ & $2831 \mathrm{~s}$ & $1036 \mathrm{~m}$ & $1580 \mathrm{~s}$ & $820 \mathrm{~s}$ & $520 \mathrm{~m}$ & $440 \mathrm{~s}$ \\
\hline$\left[\mathrm{Ru}(\mathrm{HANPT})_{3}\right] \mathrm{Cl}_{3}$ & $2831 \mathrm{~s}$ & $1030 \mathrm{~m}$ & $1590 \mathrm{~s}$ & $850 \mathrm{~s}$ & $560 \mathrm{~m}$ & $460 \mathrm{~s}$ \\
\hline$\left[\mathrm{Ru}(\mathrm{HCBPT})_{3}\right] \mathrm{Cl}_{3}$ & $2830 \mathrm{~s}$ & $1030 \mathrm{~m}$ & $1585 \mathrm{~s}$ & $852 \mathrm{~s}$ & $535 \mathrm{~s}$ & $400 \mathrm{~s}$ \\
\hline$\left[\mathrm{Ru}(\mathrm{HCBNPT})_{3}\right] \mathrm{Cl}_{3}$ & $2834 \mathrm{~m}$ & $1035 \mathrm{~m}$ & $1580 \mathrm{~s}$ & $860 \mathrm{~s}$ & $520 \mathrm{~m}$ & $430 \mathrm{~s}$ \\
\hline$\left[\mathrm{Ru}(\mathrm{HFBPT})_{3}\right] \mathrm{Cl}_{3}$ & $2841 \mathrm{~m}$ & $1040 \mathrm{~m}$ & $1600 \mathrm{~s}$ & $820 \mathrm{~s}$ & $525 \mathrm{w}$ & $410 \mathrm{~m}$ \\
\hline$\left[\mathrm{Ru}(\mathrm{HFBNPT})_{3}\right] \mathrm{Cl}_{3}$ & $2835 \mathrm{~s}$ & $1025 \mathrm{~m}$ & $1585 \mathrm{~m}$ & $860 \mathrm{~s}$ & $530 \mathrm{~m}$ & $450 \mathrm{~m}$ \\
\hline$\left[\mathrm{Ru}(\mathrm{HVPT})_{3}\right] \mathrm{Cl}_{3}$ & $2842 \mathrm{~s}$ & $1032 \mathrm{~m}$ & $1590 \mathrm{~m}$ & $850 \mathrm{~s}$ & $545 \mathrm{~m}$ & $430 \mathrm{~m}$ \\
\hline$\left[\mathrm{Ru}(\mathrm{HVNPT})_{3}\right] \mathrm{Cl}_{3}$ & $2841 \mathrm{~s}$ & $1033 \mathrm{~m}$ & $1600 \mathrm{~s}$ & $820 \mathrm{~s}$ & $560 \mathrm{~m}$ & $435 \mathrm{~s}$ \\
\hline$\left[\mathrm{Rh}(\mathrm{HAPT})_{3}\right] \mathrm{Cl}_{3}$ & $2830 \mathrm{~m}$ & $1035 \mathrm{~m}$ & $1580 \mathrm{~s}$ & $820 \mathrm{~s}$ & $520 \mathrm{~m}$ & $430 \mathrm{w}$ \\
\hline$\left[\mathrm{Rh}(\mathrm{HANPT})_{3}\right] \mathrm{Cl}_{3}$ & $2832 \mathrm{~s}$ & $1032 \mathrm{~m}$ & $1589 \mathrm{~m}$ & $840 \mathrm{~s}$ & $530 \mathrm{w}$ & $450 \mathrm{w}$ \\
\hline$\left[\mathrm{Rh}(\mathrm{HCBPT})_{3}\right] \mathrm{Cl}_{3}$ & $2831 \mathrm{~m}$ & $1032 \mathrm{~m}$ & $1580 \mathrm{~s}$ & $850 \mathrm{~s}$ & $540 \mathrm{w}$ & $445 \mathrm{w}$ \\
\hline$\left[\mathrm{Rh}(\mathrm{HCBNPT})_{3}\right] \mathrm{Cl}_{3}$ & $2836 \mathrm{~s}$ & $1035 \mathrm{~m}$ & $1585 \mathrm{~s}$ & $855 \mathrm{~s}$ & $560 \mathrm{w}$ & $418 \mathrm{~m}$ \\
\hline$\left[\mathrm{Rh}(\mathrm{HFBPT})_{3}\right] \mathrm{Cl}_{3}$ & $2841 \mathrm{~s}$ & $1038 \mathrm{~m}$ & $1600 s$ & $820 \mathrm{~s}$ & $545 \mathrm{~m}$ & $415 \mathrm{~m}$ \\
\hline$\left[\mathrm{Rh}(\mathrm{HFBNPT})_{3}\right] \mathrm{Cl}_{3}$ & $2835 \mathrm{w}$ & $1028 \mathrm{~m}$ & $1580 \mathrm{~s}$ & $860 \mathrm{~s}$ & $542 \mathrm{~m}$ & $425 \mathrm{~s}$ \\
\hline$\left[\mathrm{Rh}(\mathrm{HVPT})_{3}\right] \mathrm{Cl}_{3}$ & $2842 \mathrm{~s}$ & $1036 \mathrm{~m}$ & $1592 \mathrm{~m}$ & $845 \mathrm{~s}$ & $535 \mathrm{~m}$ & $435 \mathrm{~s}$ \\
\hline$\left[\mathrm{Rh}(\mathrm{HVNPT})_{3}\right] \mathrm{Cl}_{3}$ & $2841 \mathrm{~s}$ & $1030 \mathrm{~m}$ & $1598 \mathrm{~s}$ & $820 \mathrm{~s}$ & $532 \mathrm{~m}$ & $440 \mathrm{~m}$ \\
\hline
\end{tabular}

at $872-827 \mathrm{~cm}^{-1}$ in ligands is mainly due to the $\nu(\mathrm{C}=\mathrm{S})$ stretching vibration which shifted towards lower frequency and occurred at $860-820 \mathrm{~cm}^{-1}$ in metal complexes indicating the coordination of thione sulphur to metal atom [30]. This also described a considerable change in bond order and a metal-sulphur bond. As the $v(\mathrm{~S}-\mathrm{H})$ band also remains $\mathrm{ab}-$ sent, this confirms thione form of the ligand. In ligands as well as in complexes, the peak of $v(\mathrm{~N}-\mathrm{H})$ has been observed at $2842-2830 \mathrm{~cm}^{-1}$, which described no prominent change hence, deprotonation of ligands was not observed. Sharp and strong bands in continuous study of the spectra were observed as prominent peaks as $v(\mathrm{M}-\mathrm{N})[31], v(\mathrm{M}-\mathrm{S})[32]$ at 560-520 and at 460-400 $\mathrm{cm}^{-1}$, respectively.

\section{ELECTRONIC SPECTRA}

All of the formed complexes have been found to be in +3 oxidation state. Ruthenium(III) complexes act as paramagnetic one and rhodium(III) complexes are diamagnetic. Electronic spectral data are given in Table 3. The ground state of ruthenium(III) is ${ }^{2} \mathrm{~T}_{2 \mathrm{~g}}$ and the first excited doublet levels in order of increasing energy are ${ }^{2} \mathrm{~A}_{2 \mathrm{~g}}$ and ${ }^{2} \mathrm{~T}_{1 \mathrm{~g}}$ which are known to arise from $\mathrm{t}_{2 \mathrm{~g}}{ }^{4} \mathrm{e}_{g}^{1}$ configuration [33]. The ruthenium(III) complexes display electronic spectra with transition at $13500-14000 \mathrm{~cm}^{-1}, 17240-18300 \mathrm{~cm}^{-1}$, and $23280-$ $23800 \mathrm{~cm}^{-1}$ which may be assigned to ${ }^{2} \mathrm{~T}_{2 \mathrm{~g}} \rightarrow{ }^{4} \mathrm{~T}_{1 \mathrm{~g}},{ }^{2} \mathrm{~T}_{2 \mathrm{~g}} \rightarrow$ ${ }^{4} \mathrm{~T}_{2 \mathrm{~g}}$, and ${ }^{2} \mathrm{~T}_{2 \mathrm{~g}} \rightarrow{ }^{2} \mathrm{~A}_{2 \mathrm{~g}},{ }^{2} \mathrm{~T}_{1 \mathrm{~g}}$ in increasing order of energy. The $\mathrm{B}, \mathrm{C}$, and $10 \mathrm{Dq}$ parameters were calculated using the following equations [34]:

$$
\begin{gathered}
{ }^{2} \mathrm{~T}_{2 \mathrm{~g}}\left(\mathrm{t}^{5}\right)=0, \\
{ }^{4} \mathrm{~T}_{1 \mathrm{~g}}\left(\mathrm{t}^{4} \mathrm{e}\right)=10 \mathrm{Dq}-5 \mathrm{~B}-4 \mathrm{C}, \\
{ }^{4} \mathrm{~T}_{2 \mathrm{~g}}\left(\mathrm{t}^{4} \mathrm{e}\right)=10 \mathrm{Dq}+3 \mathrm{~B}-4 \mathrm{C}, \\
{ }^{2} \mathrm{~A}_{2 \mathrm{~g}},{ }^{2} \mathrm{~T}_{1 \mathrm{~g}}\left(\mathrm{t}^{4} \mathrm{e}\right)=10 \mathrm{Dq}-2 \mathrm{~B}-\mathrm{C} .
\end{gathered}
$$

The values of these ligand field parameters are comparable to those reported for other trivalent ruthenium complexes involving nitrogen, sulphur donor molecules [35]. The values are $c a .70-90 \%$ of the free ion values. The considerable decrease in the Racah interelectronic repulsion parameter, $\mathrm{B}$, suggests the presence of strong covalent bonding between the donor and the metal ions. The overall effect will be an increase in the observed Dq value; high Dq values 
TABLE 3: Electronic spectral bands $\left(\mathrm{cm}^{-1}\right)$ and ligand field parameters of the $\mathrm{Ru}(\mathrm{III})$ and $\mathrm{Rh}(\mathrm{III})$ complexes.

\begin{tabular}{|c|c|c|c|c|c|c|c|}
\hline Complex & $\lambda_{\max }\left(\mathrm{cm}^{-1}\right)$ & Assignments & $v_{2} / \nu_{1}$ & $10 \mathrm{Dq}\left(\mathrm{cm}^{-1}\right)$ & $\mathrm{B}\left(\mathrm{cm}^{-1}\right)$ & $\mathrm{C}\left(\mathrm{cm}^{-1}\right)$ & $\beta$ \\
\hline$\left[\mathrm{Ru}(\mathrm{HAPT})_{3}\right] \mathrm{Cl}_{3}$ & $\begin{array}{l}13700 \\
17240 \\
23600\end{array}$ & $\begin{array}{c}{ }^{2} \mathrm{~T}_{2 \mathrm{~g}} \rightarrow{ }^{4} \mathrm{~T}_{1 \mathrm{~g}}\left(\nu_{1}\right) \\
{ }^{2} \mathrm{~T}_{2 \mathrm{~g}} \rightarrow{ }^{4} \mathrm{~T}_{2 \mathrm{~g}}\left(\nu_{2}\right) \\
{ }^{2} \mathrm{~T}_{2 \mathrm{~g}} \rightarrow{ }^{2} \mathrm{~A}_{2 \mathrm{~g}^{\prime}}{ }^{2} \mathrm{~T}_{1 \mathrm{~g}}\left(\nu_{3}\right)\end{array}$ & 1.25 & 27342 & 443 & 2858 & 0.70 \\
\hline$\left[\mathrm{Ru}(\mathrm{HANPT})_{3}\right] \mathrm{Cl}_{3}$ & $\begin{array}{l}13500 \\
17260 \\
23560\end{array}$ & -do- & 1.27 & 27383 & 470 & 2883 & 0.75 \\
\hline$\left[\mathrm{Ru}(\mathrm{HCBPT})_{3}\right] \mathrm{Cl}_{3}$ & $\begin{array}{l}14060 \\
17860 \\
23600 \\
\end{array}$ & -do- & 1.27 & 27255 & 475 & 2705 & 0.76 \\
\hline$\left[\mathrm{Ru}(\mathrm{HCBNPT})_{3}\right] \mathrm{Cl}_{3}$ & $\begin{array}{l}13520 \\
17310 \\
23540\end{array}$ & -do- & 1.28 & 27352 & 474 & 2866 & 0.75 \\
\hline$\left[\mathrm{Ru}(\mathrm{HFBPT})_{3}\right] \mathrm{Cl}_{3}$ & $\begin{array}{l}13620 \\
17930 \\
23460 \\
\end{array}$ & -do- & 1.31 & 27277 & 539 & 2741 & 0.86 \\
\hline$\left[\mathrm{Ru}(\mathrm{HFBNPT})_{3}\right] \mathrm{Cl}_{3}$ & $\begin{array}{l}14000 \\
18300 \\
23580\end{array}$ & -do- & 1.30 & 27309 & 538 & 2656 & 0.86 \\
\hline$\left[\mathrm{Ru}(\mathrm{HVPT})_{3}\right] \mathrm{Cl}_{3}$ & $\begin{array}{l}13510 \\
18030 \\
23800 \\
\end{array}$ & -do- & 1.33 & 27795 & 565 & 2865 & 0.90 \\
\hline$\left[\mathrm{Ru}(\mathrm{HVNPT})_{3}\right] \mathrm{Cl}_{3}$ & $\begin{array}{l}14060 \\
18240 \\
23280\end{array}$ & -do- & 1.29 & 26875 & 523 & 2551 & 0.83 \\
\hline$\left[\mathrm{Rh}(\mathrm{HAPT})_{3}\right] \mathrm{Cl}_{3}$ & $\begin{array}{l}17600 \\
20210 \\
27170\end{array}$ & $\begin{array}{c}{ }^{1} \mathrm{~A}_{1 \mathrm{~g}} \rightarrow{ }^{3} \mathrm{~T}_{1 \mathrm{~g}} \\
{ }^{1} \mathrm{~A}_{1 \mathrm{~g}} \rightarrow{ }^{1} \mathrm{~T}_{1 \mathrm{~g}}\left(\nu_{1}\right) \\
{ }^{1} \mathrm{~A}_{1 \mathrm{~g}} \rightarrow{ }^{1} \mathrm{~T}_{2 \mathrm{~g}}\left(\nu_{2}\right)\end{array}$ & 1.34 & 21950 & 435 & 1740 & 0.60 \\
\hline$\left[\mathrm{Rh}(\mathrm{HANPT})_{3}\right] \mathrm{Cl}_{3}$ & $\begin{array}{l}17550 \\
20280 \\
27400 \\
\end{array}$ & -do- & 1.35 & 22060 & 445 & 1780 & 0.62 \\
\hline$\left[\mathrm{Rh}(\mathrm{HCBPT})_{3}\right] \mathrm{Cl}_{3}$ & $\begin{array}{l}17260 \\
20960 \\
27580 \\
\end{array}$ & -do- & 1.32 & 22615 & 413 & 1655 & 0.57 \\
\hline$\left[\mathrm{Rh}(\mathrm{HCBNPT})_{3}\right] \mathrm{Cl}_{3}$ & $\begin{array}{l}17300 \\
20220 \\
27300 \\
\end{array}$ & - do- & 1.35 & 21990 & 442 & 1770 & 0.61 \\
\hline$\left[\mathrm{Rh}(\mathrm{HFBPT})_{3}\right] \mathrm{Cl}_{3}$ & $\begin{array}{l}17400 \\
20380 \\
28020 \\
\end{array}$ & -do- & 1.37 & 22290 & 478 & 1910 & 0.66 \\
\hline$\left[\mathrm{Rh}(\mathrm{HFBNPT})_{3}\right] \mathrm{Cl}_{3}$ & $\begin{array}{l}17640 \\
20890 \\
28590 \\
\end{array}$ & -do- & 1.37 & 22815 & 481 & 1925 & 0.67 \\
\hline$\left[\mathrm{Rh}(\mathrm{HVPT})_{3}\right] \mathrm{Cl}_{3}$ & $\begin{array}{l}17650 \\
20960 \\
27590 \\
\end{array}$ & -do- & 1.32 & 22618 & 414 & 1658 & 0.58 \\
\hline$\left[\mathrm{Rh}(\mathrm{HVNPT})_{3}\right] \mathrm{Cl}_{3}$ & $\begin{array}{l}17460 \\
20880 \\
27980\end{array}$ & -do- & 1.34 & 22655 & 444 & 1775 & 0.62 \\
\hline
\end{tabular}


are usually associated with considerable electron delocalization [36]. Rhodium(III) complexes exhibit electronic spectra with transitions at $17260-17650 \mathrm{~cm}^{-1}, 20210-20960 \mathrm{~cm}^{-1}$, and $27170-28590 \mathrm{~cm}^{-1}$. These bands resemble to those of reported transitions for other hexacoordinated rhodium complexes [37]. The ground state for rhodium(III) ion is ${ }^{1} \mathrm{~A}_{1 \mathrm{~g}}$ in octahedral field, although in many instances only ${ }^{1} \mathrm{~A}_{1 \mathrm{~g}} \rightarrow$ ${ }^{1} \mathrm{~T}_{1 \mathrm{~g}}$ spin allowed ligand field transitions to be observed. These transitions correspond to the ${ }^{1} \mathrm{~A}_{1 \mathrm{~g}} \rightarrow{ }^{3} \mathrm{~T}_{1 \mathrm{~g}},{ }^{1} \mathrm{~A}_{1 \mathrm{~g}} \rightarrow$ ${ }^{1} \mathrm{~T}_{1 \mathrm{~g}}$, and ${ }^{1} \mathrm{~A}_{1 \mathrm{~g}} \rightarrow{ }^{1} \mathrm{~T}_{2 \mathrm{~g}}$, respectively, which agree well with an octahedral geometry. The B and $10 \mathrm{Dq}$ values were calculated from the positions of their electronic bands using the following equations:

$$
\begin{aligned}
& v_{1}=10 \mathrm{Dq}-4 \mathrm{~B}+\frac{86(\mathrm{~B})^{2}}{10 \mathrm{Dq}}, \\
& v_{2}=10 \mathrm{Dq}+12 \mathrm{~B}+\frac{2(\mathrm{~B})^{2}}{10 \mathrm{Dq}} .
\end{aligned}
$$

The ratios of the energies of $v_{2}$ and $v_{1}$ are in the range $1.32-1.37$. The $B$ values are $57-67 \%$ of the free ion value. The decrease in $B$ values from the free ion value suggests that there is a considerable orbital overlap with strong covalency in the metal ligand $\sigma$ bond [38].

\section{MAGNETIC MOMENTS}

The room temperature magnetic moments of all the ruthenium(III) thiosemicarbazone complexes lie in the range 1.08-1.90 B.M., which are expected to be lower than the predicted value of 2.10 B.M. This lowering may occur due to the presence of lower symmetry ligand fields, metal-metal interactions, or extensive electron delocalization in species [39]. Rhodium(III) complexes are diamagnetic and, as expected, this is again consistent with octahedral geometry of nitrogen and sulphur atoms producing a strong field [40].

\section{1. ${ }^{1} \mathrm{H}$ and ${ }^{13} \mathrm{CNMR}$}

Coordination of thiosemicarbazones in the rhodium(III) complexes are further confirmed by ${ }^{1} \mathrm{H}$ and ${ }^{13} \mathrm{C}$ NMR spectra (see Table 4). The resonance for methoxy protons appeared as a singlet at $\delta 3.65 \mathrm{ppm}$ in ligands and in complexes no significant change was observed. Significant azomethine proton signal, due to $\mathrm{CH}=\mathrm{N}$, was observed at $\delta 8.02-9.02 \mathrm{ppm}$ region as a multiplet in ligands, and in complexes it has shown a change as a downfield shift and occurred at $\delta 8.20$ $9.20 \mathrm{ppm}$, indicating involvement of nitrogen in coordination. The proton peak of $\mathrm{N}-\mathrm{H}$ group at $\delta 10.6-11.2 \mathrm{ppm}$ remains the same in the ligands, and in the complexes it suggested that deprotonation do not occur and it has also shown keto form of the ligands. The multiplets as strong bands in region $\delta 6.2-8.2 \mathrm{ppm}$ were assigned to aromatic ring protons, which also shifted downfield in the complexes.

The ${ }^{13} \mathrm{C}$ NMR spectra revealed the presence of expected number of signals corresponding to different types of carbon atoms present in the compounds. In ligands as well as in complexes, $-\mathrm{OCH}_{3}$ group absorbs at $\delta 65.0-65.2 \mathrm{ppm}$ and at $\delta 65.5-65.6 \mathrm{ppm}$ slightly downfield to the methyl group carbon due to the deshielding of the directly attached electronegative oxygen atom. No change on complexation to this group occurs. The spectra of the ligands exhibit a strong band at $\delta 179.2-180.2 \mathrm{ppm}$ and are assigned as $\mathrm{C}=\mathrm{S}$ group. This band undergoes upfield shift of $\delta 7.2-7.4 \mathrm{ppm}$ and occurs at $\delta 171.9-172.8 \mathrm{ppm}$. This has shown involvement of thione sulphur in coordination. The signals due to azomethine carbon occurred at $\delta 162.3-165.2 \mathrm{ppm}$ as downfield peak, and on complexation they have shown shift to $\delta 160.5-$ $163.0 \mathrm{ppm}$ due to the resonance and also have given proof that nitrogen is involved in coordination.

\section{THERMAL STUDIES}

The TGA data reveal that there is a good agreement with the formulae as suggested from the elemental analyses. The first mass loss occurs within the temperature range 190$300^{\circ} \mathrm{C}$, which corresponds to the removal of three chloride ions of the outer sphere as $\mathrm{HCl}$. The number of chelate rings as well as the type of chelate rings around metal ions play an important role in the thermal stability and degradation of the complexes. Furthermore, it is known that the electronegativity and atomic radius of the central metal also affect the thermal stability. No endothermic peak has been observed, indicating absence of water molecule. Thermal investigations of $\left[\mathrm{Ru}(\mathrm{HAPT})_{3}\right] \mathrm{Cl}_{3}$ support the removal of the organic part of the ligand as PhNHCS fragments in the temperature range $320-360^{\circ} \mathrm{C}$. The third step corresponds to the removal of the three molecules of $\mathrm{C}_{7} \mathrm{H}_{5} \mathrm{OCH}_{3}$ at temperature range $400-480^{\circ} \mathrm{C}$. Final decomposition leaves a mixed residue of $\mathrm{Ru}_{2} \mathrm{O}_{3}-\mathrm{RuO}_{2}$ at $680-695^{\circ} \mathrm{C}$. The same decomposition pattern was observed for other complexes of ruthenium and rhodium leaving residues of $\mathrm{RuO}_{2}$ and $\mathrm{Rh}_{2} \mathrm{O}_{3}$, respectively, in the temperature range $710-750^{\circ} \mathrm{C}$ like a carbonaceous matter.

\section{ANTIBACTERIAL STUDIES}

The results (Table 5) exhibit that complexes show moderate activity against Bacillus subtilis and Pseudomonos aeruginosa. The toxicity of the complexes was found better than parent ligand owing to the chelation theory of Tweedy [41]. The $\left[\mathrm{Ru}(\mathrm{HVPT})_{3}\right] \mathrm{Cl}_{3},\left[\mathrm{Ru}(\mathrm{HAPT})_{3}\right] \mathrm{Cl}_{3}$, and $\left[\mathrm{Ru}(\mathrm{HVNPT})_{3}\right] \mathrm{Cl}_{3}$ exhibited higher toxicity; this is due to the presence of electron donating group $\left(\mathrm{OCH}_{3}\right)$ in these complexes while in the same complexes of rhodium better toxicity was also observed. The variation in the toxicity of different complexes against various organisms depends either on the impermeability of the cells of the microbes or differences in ribosome in microbial cells [42]. The enhanced effect of complexes due to chelation could increase the lipophilicity of the central metal atom, which favours the permeation through the lipid layers of the cell wall. On the other hand, the mode of action of the compounds may involve the formation of hydrogen bonds through azomethine group of the complexes with the active centers of cell constituents resulting in the interference with normal cell process. Besides, antibacterial activity could not reach the affectivity of the streptomycin. On the basis of 
TABLE 4: NMR spectral data $(\delta, \mathrm{ppm})$ of the thiosemicarbazones and their rhodium(III) complexes.

\begin{tabular}{|c|c|c|c|c|c|c|c|}
\hline \multirow{2}{*}{ Compounds } & \multicolumn{4}{|c|}{${ }^{1} \mathrm{H}-$} & \multicolumn{3}{|c|}{${ }^{13} \mathrm{C}-$} \\
\hline & $\delta(\mathrm{CH}=\mathrm{N})$ & $\delta(\mathrm{N}-\mathrm{H})$ & $\delta(\mathrm{Ar}-\mathrm{H})$ & $\delta\left(\mathrm{OCH}_{3}\right)$ & $\delta(\mathrm{C}=\mathrm{N})$ & $\delta(\mathrm{C}=\mathrm{S})$ & $\delta\left(\mathrm{O}-\mathrm{CH}_{3}\right)$ \\
\hline HAPT & $8.02(\mathrm{~s})$ & $10.9(\mathrm{~s})$ & $6.2-7.0(\mathrm{~m})$ & $3.62(\mathrm{~s})$ & 162.3 & 179.2 & 65.0 \\
\hline HANPT & $8.06(\mathrm{~s})$ & $11.0(\mathrm{~s})$ & $6.2-7.8(\mathrm{~m})$ & $3.65(\mathrm{~s})$ & 162.6 & 179.6 & 65.2 \\
\hline НСBPT & $8.08(\mathrm{~s})$ & $11.0(\mathrm{~s})$ & $6.2-7.6(\mathrm{~m})$ & - & 163.2 & 180.0 & - \\
\hline HCBNPT & $8.06(\mathrm{~s})$ & $11.2(\mathrm{~s})$ & $6.2-7.8(\mathrm{~m})$ & - & 163.8 & 180.3 & - \\
\hline HFBPT & $9.00(\mathrm{~s})$ & $10.9(\mathrm{~s})$ & $6.3-7.2(\mathrm{~m})$ & - & 165.0 & 179.8 & - \\
\hline HFBNPT & $9.02(\mathrm{~s})$ & $11.1(\mathrm{~s})$ & $6.6-8.0(\mathrm{~m})$ & - & 165.2 & 180.2 & - \\
\hline HVPT & $9.02(\mathrm{~s})$ & $11.0(\mathrm{~s})$ & $6.8-8.0(\mathrm{~m})$ & $3.60(\mathrm{~s})$ & 164.2 & 179.5 & 65.0 \\
\hline HVNPT & $8.08(\mathrm{~s})$ & $11.2(\mathrm{~s})$ & $6.2-7.8(\mathrm{~m})$ & $3.61(\mathrm{~s})$ & 163.9 & 180.2 & 65.2 \\
\hline$\left[\mathrm{Rh}(\mathrm{HAPT})_{3}\right] \mathrm{Cl}_{3}$ & $8.20(\mathrm{~s})$ & $10.6(\mathrm{~s})$ & $6.4-7.2(\mathrm{~m})$ & $3.65(\mathrm{~s})$ & 160.5 & 171.9 & 65.5 \\
\hline$\left[\mathrm{Rh}(\mathrm{HANPT})_{3}\right] \mathrm{Cl}_{3}$ & $8.28(\mathrm{~s})$ & $10.9(\mathrm{~s})$ & $6.5-7.6(\mathrm{~m})$ & $3.66(\mathrm{~s})$ & 160.8 & 172.2 & 65.5 \\
\hline$\left[\mathrm{Rh}(\mathrm{HCBPT})_{3}\right] \mathrm{Cl}_{3}$ & $8.26(\mathrm{~s})$ & $11.1(\mathrm{~s})$ & $6.6-7.9(\mathrm{~m})$ & - & 162.0 & 172.6 & - \\
\hline$\left[\mathrm{Rh}(\mathrm{HCBNPT})_{3}\right] \mathrm{Cl}_{3}$ & $9.20(\mathrm{~s})$ & $11.0(\mathrm{~s})$ & $6.8-8.2(\mathrm{~m})$ & - & 162.6 & 172.8 & - \\
\hline$\left[\mathrm{Rh}(\mathrm{HFBPT})_{3}\right] \mathrm{Cl}_{3}$ & $9.18(\mathrm{~s})$ & $10.9(\mathrm{~s})$ & $6.9-8.0(\mathrm{~m})$ & - & 163.0 & 172.6 & - \\
\hline$\left[\mathrm{Rh}(\mathrm{HFBNPT})_{3}\right] \mathrm{Cl}_{3}$ & $9.20(\mathrm{~s})$ & $11.0(\mathrm{~s})$ & $6.6-8.0(\mathrm{~m})$ & - & 163.0 & 172.8 & - \\
\hline$\left[\mathrm{Rh}(\mathrm{HVPT})_{3}\right] \mathrm{Cl}_{3}$ & $9.16(\mathrm{~s})$ & $11.2(\mathrm{~s})$ & $6.9-7.2(\mathrm{~m})$ & $3.62(\mathrm{~s})$ & 161.6 & 172.3 & 65.6 \\
\hline$\left[\mathrm{Rh}(\mathrm{HVNPT})_{3}\right] \mathrm{Cl}_{3}$ & $8.20(\mathrm{~s})$ & $11.0(\mathrm{~s})$ & $6.4-7.8(\mathrm{~m})$ & $3.61(\mathrm{~s})$ & 161.2 & 172.8 & 65.6 \\
\hline
\end{tabular}

TABle 5: Antibacterial screening data of thiosemicarbazones and their $\mathrm{Ru}(\mathrm{III})$ and $\mathrm{Rh}(\mathrm{III})$ complexes.

\begin{tabular}{|c|c|c|c|c|}
\hline \multirow{3}{*}{ Compounds } & \multicolumn{4}{|c|}{ Inhibition zone $\left(\mu \mathrm{gmL}^{-1}\right)$} \\
\hline & \multicolumn{2}{|c|}{ Bacillus subtilis } & \multicolumn{2}{|c|}{ Pseudomonos aeruginosa } \\
\hline & 500 & 1000 & 500 & 1000 \\
\hline HAPT & 7 & 9 & 8 & 9 \\
\hline$\left[\mathrm{Ru}(\mathrm{HAPT})_{3}\right] \mathrm{Cl}_{3}$ & 14 & 17 & 13 & 16 \\
\hline$\left[\mathrm{Rh}(\mathrm{HAPT})_{3}\right] \mathrm{Cl}_{3}$ & 10 & 12 & 9 & 12 \\
\hline HANPT & 7 & 10 & 7 & 11 \\
\hline$\left[\mathrm{Ru}(\mathrm{HANPT})_{3}\right] \mathrm{Cl}_{3}$ & 12 & 16 & 11 & 16 \\
\hline$\left[\mathrm{Rh}(\mathrm{HANPT})_{3}\right] \mathrm{Cl}_{3}$ & 10 & 14 & 10 & 13 \\
\hline НСВРТ & 6 & 10 & 6 & 9 \\
\hline$\left[\mathrm{Ru}(\mathrm{HCBPT})_{3}\right] \mathrm{Cl}_{3}$ & 13 & 16 & 12 & 16 \\
\hline$\left[\mathrm{Rh}(\mathrm{HCBPT})_{3}\right] \mathrm{Cl}_{3}$ & 11 & 16 & 11 & 14 \\
\hline HCBNPT & 7 & 10 & 7 & 11 \\
\hline$\left[\mathrm{Ru}(\mathrm{HCBNPT})_{3}\right] \mathrm{Cl}_{3}$ & 14 & 18 & 15 & 19 \\
\hline$\left[\mathrm{Rh}(\mathrm{HCBNPT})_{3}\right] \mathrm{Cl}_{3}$ & 12 & 14 & 13 & 15 \\
\hline HFBPT & 7 & 9 & 6 & 10 \\
\hline$\left[\mathrm{Ru}(\mathrm{HFBPT})_{3}\right] \mathrm{Cl}_{3}$ & 13 & 16 & 13 & 17 \\
\hline$\left[\mathrm{Rh}(\mathrm{HFBPT})_{3}\right] \mathrm{Cl}_{3}$ & 10 & 13 & 10 & 12 \\
\hline HFBNPT & 6 & 10 & 6 & 9 \\
\hline$\left[\mathrm{Ru}(\mathrm{HFBNPT})_{3}\right] \mathrm{Cl}_{3}$ & 14 & 17 & 13 & 16 \\
\hline$\left[\mathrm{Rh}(\mathrm{HFBNPT})_{3}\right] \mathrm{Cl}_{3}$ & 11 & 14 & 11 & 15 \\
\hline HVPT & 9 & 12 & 9 & 11 \\
\hline$\left[\mathrm{Ru}(\mathrm{HVPT})_{3}\right] \mathrm{Cl}_{3}$ & 14 & 17 & 14 & 16 \\
\hline$\left[\mathrm{Rh}(\mathrm{HVPT})_{3}\right] \mathrm{Cl}_{3}$ & 11 & 14 & 11 & 13 \\
\hline HVNPT & 8 & 10 & 9 & 11 \\
\hline$\left[\mathrm{Ru}(\mathrm{HVNPT})_{3}\right] \mathrm{Cl}_{3}$ & 15 & 18 & 14 & 18 \\
\hline$\left[\mathrm{Rh}(\mathrm{HVNPT})_{3}\right] \mathrm{Cl}_{3}$ & 12 & 15 & 12 & 14 \\
\hline Streptomycin & 17 & 18 & 21 & 22 \\
\hline
\end{tabular}


the above studies, the structures in Figure 2 may be formulated for the complexes.

\section{ACKNOWLEDGMENT}

The first author is grateful to the University Grants Commission, New Delhi, India, for financial assistance (project no. F.12-37/2003 (SR)).

\section{REFERENCES}

[1] D. Mishra, S. Naskar, M. G. B. Drew, and S. K. Chattopadhyay, "Synthesis, spectroscopic and redox properties of some ruthenium(II) thiosemicarbazone complexes: structural description of four of these complexes," Inorganica Chimica Acta, vol. 359, no. 2, pp. 585-592, 2006.

[2] J. S. Casas, M. S. García-Tasende, and J. Sordo, "Main group metal complexes of semicarbazones and thiosemicarbazones. A structural review," Coordination Chemistry Reviews, vol. 209, no. 1, pp. 197-261, 2000.

[3] S. Padhyé and G. B. Kauffman, "Transition metal complexes of semicarbazones and thiosemicarbazones," Coordination Chemistry Reviews, vol. 63, pp. 127-160, 1985.

[4] I. Pal, F. Basuli, and S. Bhattacharya, "Thiosemicarbazone complexes of the platinum metals. A story of variable coordination modes," Proceedings of the Indian Academy of Sciences: Chemical Sciences, vol. 114, no. 4, pp. 255-268, 2002.

[5] M. Belicchi Ferrari, S. Capacchi, G. Pelosi, et al., "Synthesis, structural characterization and biological activity of helicin thiosemicarbazone monohydrate and a copper(II) complex of salicylaldehyde thiosemicarbazone," Inorganica Chimica Acta, vol. 286, no. 2, pp. 134-141, 1999.

[6] S. Dutta, F. Basuli, S.-M. Peng, G.-H. Lee, and S. Bhattacharya, "Synthesis, structure and redox properties of some thiosemicarbazone complexes of rhodium," New Journal of Chemistry, vol. 26, no. 11, pp. 1607-1612, 2002.

[7] A. K. El-Sawaf, D. X. West, F. A. El-Saied, and R. M. ElBahnasawy, "Synthesis, magnetic and spectral studies of iron(III), cobalt(II, III), nickel(II), copper(II) and zinc(II) complexes of 4-formylantipyrine $\mathrm{N}(4)$-antipyrinylthiosemicarbazone," Transition Metal Chemistry, vol. 23, no. 5, pp. 649-655, 1998.

[8] S. Purohit, A. P. Koley, L. S. Prasad, P. T. Manoharan, and S. Ghosh, "Chemistry of molybdenum with hard-soft donor ligands. 2. Molybdenum(VI), -(V), and -(IV) oxo complexes with tridentate Schiff base ligands," Inorganic Chemistry, vol. 28, no. 19, pp. 3735-3742, 1989.

[9] D. X. West, J. K. Swearingen, J. Valdés-Martínez, et al., "Spectral and structural studies of iron(III), cobalt(II,III) and nickel(II) complexes of 2-pyridineformamide N(4)-methylthiosemicarbazone," Polyhedron, vol. 18, no. 22, pp. 29192929, 1999.

[10] Z. Afrasiabi, E. Sinn, J. Chen, et al., "Appended 1,2-naphthoquinones as anticancer agents 1: synthesis, structural, spectral and antitumor activities of ortho-naphthaquinone thiosemicarbazone and its transition metal complexes," Inorganica Chimica Acta, vol. 357, no. 1, pp. 271-278, 2004.

[11] D. Kovala-Demertzi, J. R. Miller, N. Kourkoumelis, S. K. Hadjikakou, and M. A. Demertzis, "Palladium(II) and platinum(II) complexes of pyridine-2-carbaldehyde thiosemicarbazone with potential biological activity. Synthesis, structure and spectral properties. Extended network via hydrogen bond linkages of $[\mathrm{Pd}(\mathrm{PyTsc}) \mathrm{Cl}]$," Polyhedron, vol. 18, no. 7, pp. 1005-1013, 1999.

[12] N. K. Singh and S. B. Singh, "Synthesis, characterization and biological properties of manganese(II), cobalt(II), nickel(II), copper(II), zinc(II), chromium(III) and iron(III) complexes with a new thiosemicarbazide derivative," Indian Journal of Chemistry, vol. 40, no. 10, pp. 1070-1075, 2001.

[13] R. K. Agarwal, L. Singh, and D. K. Sharma, "Synthesis, spectral, and biological properties of copper(II) complexes of thiosemicarbazones of Schiff bases derived from 4-aminoantipyrine and aromatic aldehydes," Bioinorganic Chemistry and Applications, vol. 2006, Article ID 59509, 10 pages, 2006.

[14] J. García-Tojal, L. Lezama, J. L. Pizarro, M. Insausti, M. I. Arriortua, and T. Rojo, "Spectroscopic and magnetic properties of copper(II) complexes derived from pyridine-2carbaldehyde thiosemicarbazone. Structures of $\left[\mathrm{Cu}\left(\mathrm{NO}_{3}\right)-\right.$ $\left.\left(\mathrm{C}_{7} \mathrm{H}_{8} \mathrm{~N}_{4} \mathrm{~S}\right)\left(\mathrm{H}_{2} \mathrm{O}\right)\right]\left(\mathrm{NO}_{3}\right)$ and $\left[\left\{\mathrm{Cu}(\mathrm{NCS})\left(\mathrm{C}_{7} \mathrm{H}_{7} \mathrm{~N}_{4} \mathrm{~S}\right)\right\}_{2}\right]$," Polyhedron, vol. 18, no. 27, pp. 3703-3711, 1999.

[15] E. Labisbal, K. D. Haslow, A. Sousa-Pedrares, J. ValdésMartínez, S. Hernández-Ortega, and D. X. West, "Copper(II) and nickel(II) complexes of 5-methyl-2-hydroxyacetophenone N(4)-substituted thiosemicarbazones," Polyhedron, vol. 22, no. 20, pp. 2831-2837, 2003.

[16] R. M. El-Shazly, G. A. A. Al-Hazmi, S. E. Ghazy, M. S. El-Shahawi, and A. A. El-Asmy, "Spectroscopic, thermal and electrochemical studies on some nickel(II) thiosemicarbazone complexes," Spectrochimica Acta-Part A: Molecular and Biomolecular Spectroscopy, vol. 61, no. 1-2, pp. 243-252, 2005.

[17] S. I. Mostafa, A. A. El-Asmy, and M. S. El-Shahawi, "Ruthenium(II) 2-hydroxybenzophenone N(4)-substituted thiosemicarbazone complexes," Transition Metal Chemistry, vol. 25, no. 4, pp. 470-473, 2000.

[18] S. K. Chattopadhyay and S. A. Ghosh, "A study of Ru(II) complexes of some selected $\mathrm{N}-\mathrm{S}$ donors," Inorganica Chimica Acta, vol. 131, no. 1, pp. 15-20, 1987.

[19] K. Mukkanti and R. P. Singh, "Complexes of platinum, rhodium, iridium and ruthenium with a thiosemicarbazone derived from thiophene-2-carboxaldehyde," Transition Metal Chemistry, vol. 12, no. 4, pp. 299-301, 1987.

[20] P. K. Sinha, J. Chakravarty, and S. Bhattacharya, "Synthesis, characterization, redox properties and reactivities of a group of phenolato complexes of ruthenium(III)," Polyhedron, vol. 16 , no. 1, pp. 81-87, 1997.

[21] J. Chakravarty and S. Bhattacharya, "Synthesis, characterization, electron-transfer properties and reactivities of a group of ruthenium(II) complexes with $\mathrm{RuN}_{2} \mathrm{P}_{2} \mathrm{X}_{2}(\mathrm{X}=\mathrm{Cl}, \mathrm{Br})$ coordination spheres," Polyhedron, vol. 13, no. 18, pp. 2671-2678, 1994.

[22] S. K Chattopadhyay, M. Hossain, S. Ghosh, and A. K. Guha, "Ligational behaviour of two biologically active $\mathrm{N}-\mathrm{S}$ donors towards cobalt(III), iron(III), iron(II) and rhodium(III)," Transition Metal Chemistry, vol. 15, no. 6, pp. 473-477, 1990.

[23] C. Jayabalakrishnan, R. Karvembu, and K. Natarajan, "Synthesis, characterisation, catalytic, and biocidal studies of ruthenium(III) complexes with thiosemicarbazones of $\beta$ diketoesters," Synthesis and Reactivity in Inorganic, MetalOrganic, and Nano-Metal Chemistry, vol. 32, no. 6, pp. 10991113, 2002.

[24] C. H. Collins and P. M. Lyne, Microbial Methods, University Park Press, Baltimore, Md, USA, 1970. 
[25] P. Sengupta, R. Dinda, and S. Ghosh, "Ruthenium(II) complexes of NSO donor ligands in the form of ring-substituted 4-phenyl-thiosemicarbazones of salicylaldehyde and ohydroxyacetophenone," Transition Metal Chemistry, vol. 27, no. 6, pp. 665-667, 2002.

[26] A. I. Vogel, A Textbook of Quantitative Inorganic Analyses, Longmans, London, UK, 4th edition, 1986.

[27] K. P. Deepa and K. K. Aravindakshan, "Synthesis, characterization and thermal studies of thiosemicarbazones of N-methyland N-ethylacetoacetanilide," Synthesis and Reactivity in Inorganic, Metal-Organic, and Nano-Metal Chemistry, vol. 30, no. 8, pp. 1601-1616, 2000.

[28] M. Joseph, A. Sreekanth, V. Suni, and M. R. P. Kurup, "Spectral characterization of iron(III) complexes of 2-benzoylpyridine N(4)-substituted thiosemicarbazones," Spectrochimica ActaPart A: Molecular and Biomolecular Spectroscopy, vol. 64, no. 3, pp. 637-641, 2006.

[29] O. E. Offiong and S. Martelli, "Stereochemistry and antitumour activity of platinum metal complexes of 2acetylpyridine thiosemicarbazones," Transition Metal Chemistry, vol. 22, no. 3, pp. 263-269, 1997.

[30] S. Chandra and L. K. Gupta, "EPR, mass, IR, electronic, and magnetic studies on copper(II) complexes of semicarbazones and thiosemicarbazones," Spectrochimica Acta-Part A: Molecular and Biomolecular Spectroscopy, vol. 61, no. 1-2, pp. 269275, 2005.

[31] V. K. Sharma, S. Srivastava, and A. Srivastava, "Novel coordination complexes of the trivalent ruthenium, rhodium and iridium with hydrazones derived from Isatin hydrazide and various aldehydes with spectral and biological characterization," Polish Journal of Chemistry, vol. 80, no. 3, pp. 387-396, 2006.

[32] A. S. El-Tabl and M. I. Ayad, "Investigation of thiosemicarbazones as chelating agents. Synthesis and spectroscopic characterization of some new ruthenium(III) complexes," Synthesis and Reactivity in Inorganic, Metal-Organic, and Nano-Metal Chemistry, vol. 33, no. 3, pp. 369-385, 2003.

[33] G. Venkatachalam, S. Maheswaran, and R. Ramesh, "Synthesis, spectra, redox property and catalytic activity of ruthenium(III) Schiff base complexes," Indian Journal of Chemistry, vol. 44, no. 4, pp. 705-709, 2005.

[34] A. B. P. Lever, "Electronic spectra of $\mathrm{d}^{\mathrm{n}}$ ions," in Inorganic Electronic Spectroscopy, pp. 376-611, Elsevier, Amsterdam, The Netherlands, 2nd edition, 1984.

[35] S. Chandra, "Pd(II), Pt(II), Rh(III), Ir(III) and Ru(III) complexes of n-pentyl and n-hexyl ketone thiosemicarbazones," Synthesis and Reactivity in Inorganic, Metal-Organic, and Nano-Metal Chemistry, vol. 22, no. 10, pp. 1565-1579, 1992.

[36] C. K. Jorgensen, Absorption Spectra and Chemical Bonding in Complexes, Pergamon Press, London, UK, 1964.

[37] S. Chandra and R. Singh, "Pd(II), Pt(II), Rh(III), Ir(III) and $\mathrm{Ru}(\mathrm{III})$ complexes of some nitrogen -oxygen donor ligands," Indian Journal of Chemistry, vol. 27A, pp. 417-420, 1988.

[38] A. El-Dissouky, A. Kasem, and A. Z. El-Sonbati, "Synthesis and characterization of some octahedral transition metal complexes with phenyl-2-picolylketone thiosemicarbazone," Transition Metal Chemistry, vol. 12, no. 6, pp. 500-503, 1987.

[39] F. A. El-Saied, R. M. El-Bahnasawy, M. Abdel-Azeem, and A. K. El-Sawaf, "Synthesis, characterization and electrochemical properties of $\beta$-diketone complexes of ruthenium(III)," Polyhedron, vol. 13, no. 11, pp. 1781-1785, 1994.
[40] V. K. Sharma and S. K. Sengupta, "Synthesis and spectroscopic studies on ruthenium (III) and rhodium (III) derivatives with thiohydantoins," Synthesis and Reactivity in Inorganic, MetalOrganic, and Nano-Metal Chemistry, vol. 23, no. 3, pp. 401418, 1993.

[41] T. D. Thangadurai and K. Natarajan, "Synthesis and characterisation of ruthenium(III) complexes containing dibasic tetradentate Schiff bases," Indian Journal of Chemistry, vol. 41, no. 4, pp. 741-745, 2002.

[42] S. K. Sengupta, O. P. Pandey, B. K. Srivastava, and V. K. Sharma, "Synthesis, structural and biochemical aspects of titanocene and zirconocene chelates of acetylferrocenyl thiosemicarbazones," Transition Metal Chemistry, vol. 23, no. 4, pp. 349-353, 1998. 


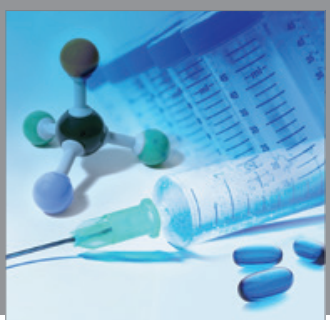

International Journal of

Medicinal Chemistry

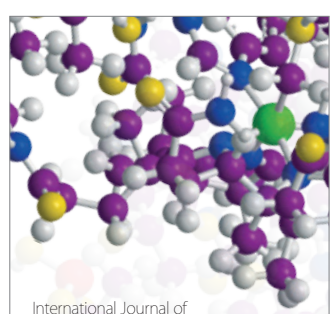

Carbohydrate Chemistry

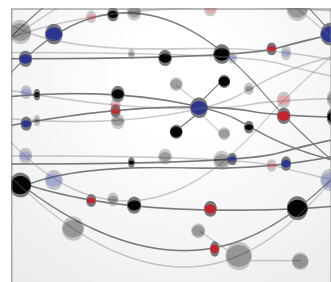

The Scientific World Journal
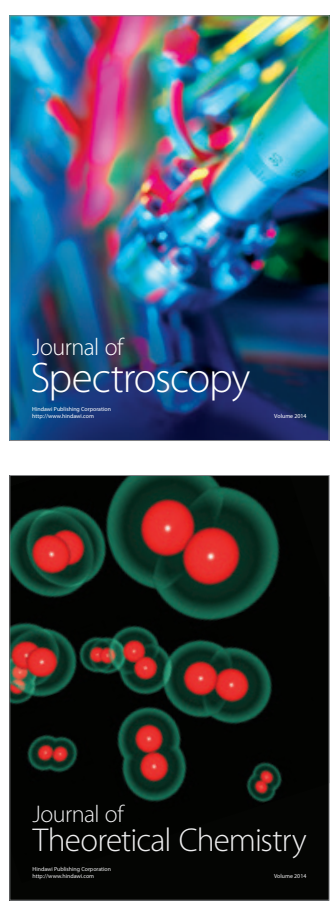
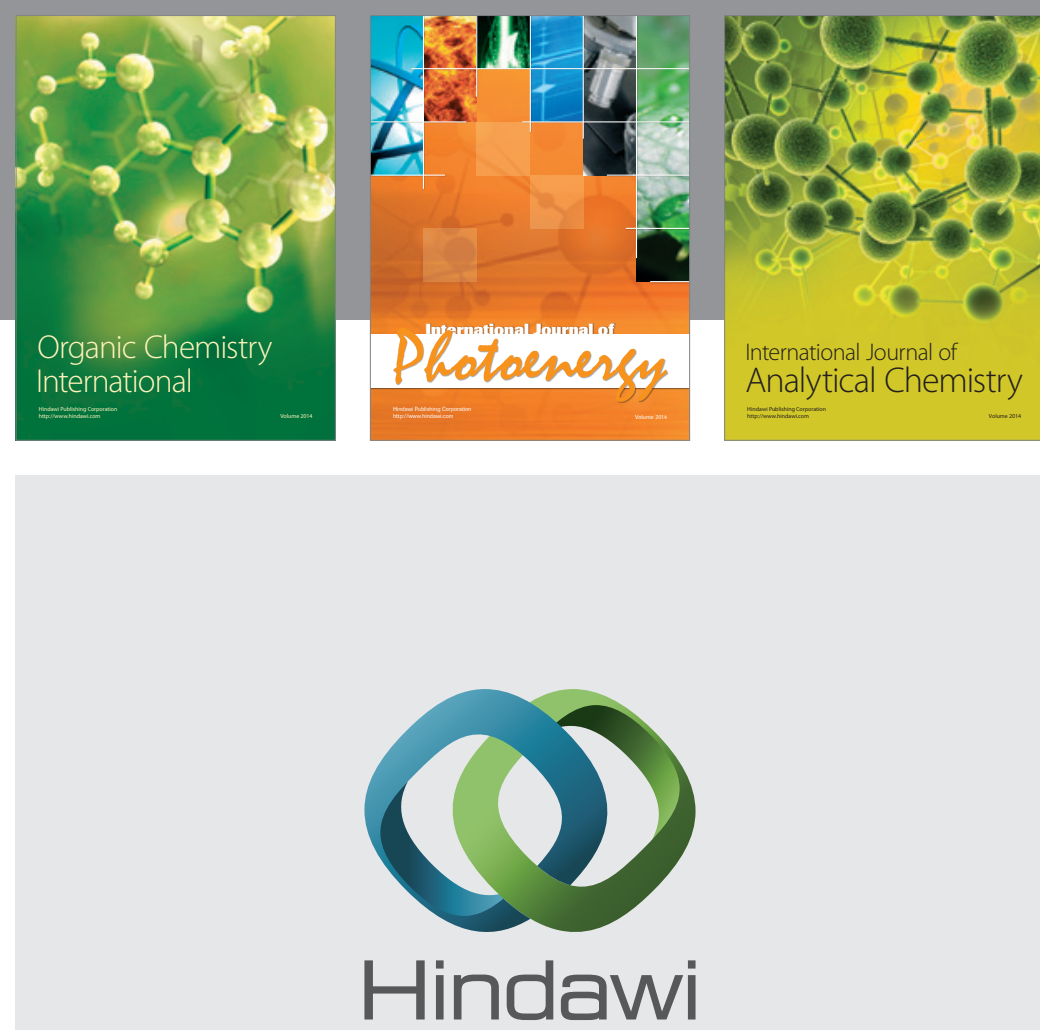

Submit your manuscripts at

http://www.hindawi.com
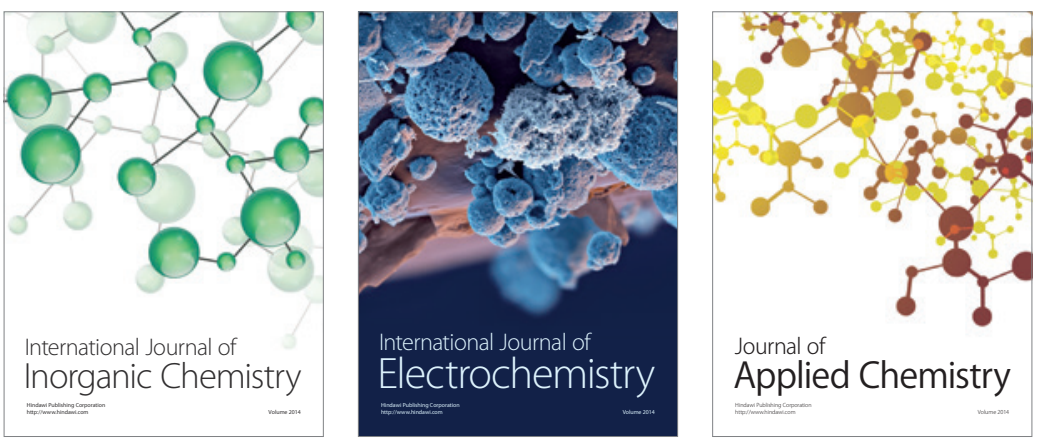

Journal of

Applied Chemistry
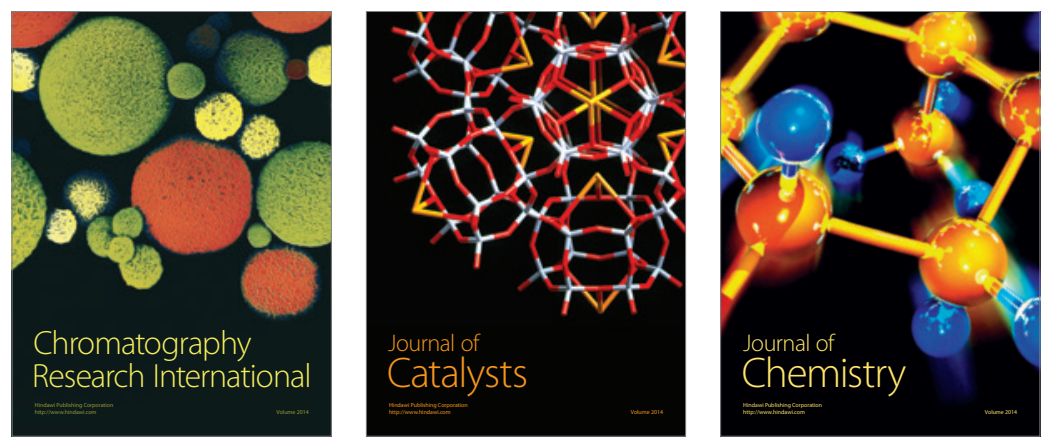
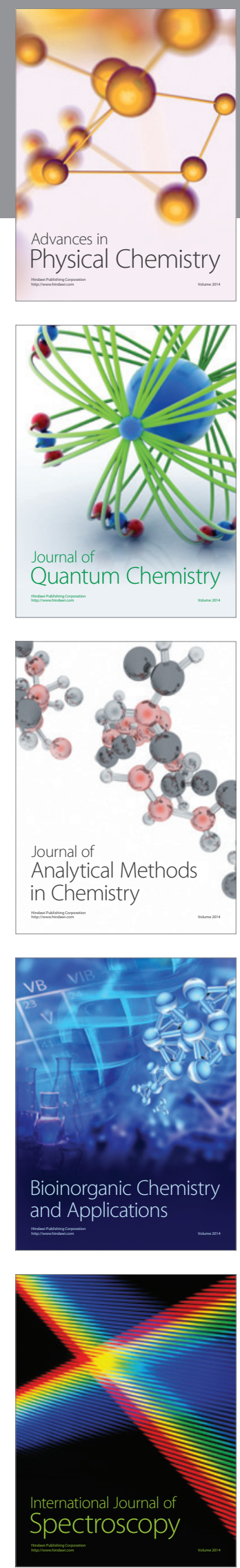